\title{
Cytotoxic effects of adenovirus- and lentivirus- mediated expression of Drosophila melanogaster deoxyribonucleoside kinase on Bcap37 breast cancer cells
}

\author{
NIANQU ZHANG ${ }^{1 *}$, XIAOSHEN DONG $^{1 *}$, YIQUN SUN $^{3}$, XIAOPENG CAI $^{1}$, \\ CAIWEI ZHENG ${ }^{4}, \mathrm{ANNING} \mathrm{HE}^{5}, \mathrm{KE} \mathrm{XU}^{2}$ and XINYU ZHENG ${ }^{1,5}$ \\ ${ }^{1}$ Department of Surgical Oncology, Department of General Surgery, ${ }^{2}$ Department of Radiology, \\ First Affiliated Hospital, China Medical University, Shenyang; ${ }^{3}$ Department of Burns, General Hospital \\ of Benxi Iron and Steel Company, Benxi, P.R. China; ${ }^{4}$ Brandeis University, Waltham, MA, USA; \\ ${ }^{5}$ Laboratory 1 , Cancer Institute, China Medical University, Shenyang, P.R. China
}

Received September 25, 2012; Accepted November 2, 2012

DOI: $10.3892 /$ or.2012.2194

\begin{abstract}
Gene transfer using different viral vectors has demonstrated different antitumor effects in suicide gene therapy. In the present study, in order to optimize the efficacy of replication-defective adenoviral and lentiviral vectors for gene therapy, RT-PCR was used to evaluate the expression of Drosophila melanogaster deoxyribonucleoside kinase $(D m-d N K)$ in the Bcap37 human breast cancer cell line, dThd was used to determine the activity of $D m-d N K$, cell cytotoxicity was evaluated by MTT assay and cell proliferation was assessed using a hemocytometer. Moreover, apoptosis induction was evaluated by the Annexin V-FITC-labeled FACS method. Furthermore, BALB/C nude mice bearing tumors were treated with $D m-d N K$ mediated with the pyrimidine nucleoside analog, brivudine [BVDU, (E)-5-(2-bromovinyl)-2'-deoxyuridine]. Our results indicated that the gene expression of $D m-d N K$ transfected by adenoviral and lentiviral vectors may be detected and that its long-term activity may be retained. Both vectors containing the $D m-d N K$ gene revealed high cytotoxicity and sensitized cell apoptosis from the BVDU prodrug. In tumor models, lentivirus-mediated gene therapy significantly inhibited the growth of tumors compared with adenovirus-mediated
\end{abstract}

Correspondence to: Dr Xinyu Zheng, Department of Surgical Oncology, First Affiliated Hospital, China Medical University, 155 North Nanjing Street, Shenyang 110001, P.R. China

E-mail: xyzheng@mail.cmu.edu.cn

Dr Ke Xu, Department of Radiology, First Affiliated Hospital, China Medical University, 155 North Nanjing Street, Shenyang 110001, P.R. China

E-mail: kexu@vip.sina.com

${ }^{*}$ Contributed equally

Key words: Drosophila melanogaster deoxyribonucleoside kinase, adenovirus, lentivirus, nucleoside analog, suicide gene gene therapy. Although adenovirus- and lentivirus-transduced $D m-d N K$ reveal strong treatment efficacy in vitro, the latter has great potential due to the long-term expression of the therapeutic gene in vivo.

\section{Introduction}

Breast cancer is a major life-threating disease in females worldwide (1). Despite current advances in chemotherapy, the outcome for patients with metastatic breast cancer remains unsatisfactory. Certain patients refuse to accept this type of treatment due to the severe side-effects. Therefore, a more effective chemotherapeutic combination treatment associated with manageable toxicity is highly desired. As a potential strategy, suicide gene therapy is a promising therapeutic approach for improving treatment efficacy and the application of nucleoside kinases as suicide genes in gene therapy has been extensively studied. The basic concept is to transduce cells with the gene encoding herpes simplex virus type I thymidine kinase (HSV-TK) and subsequently expose the cells to a ganciclovir (GCV) prodrug. To date, this enzyme-directed prodrug therapeutic combination with HSV-TK/GCV has been approved for phase III clinical trials.

With the development of suicide gene therapy, a deoxyribonucleoside kinase of Drosophila melanogaster (Dm-dNK) in cultured Drosophila S-2 cells has been introduced (2). Compared with HSV-TK and other enzymes, $D m-d N K$ has a remarkably broad substrate specificity and a higher catalytic rate with the capacity to phosphorylate all 4 deoxyribonucleosides when transferred to human cells. The isolated kinase has a preference for a pyrimidine nucleoside, such as brivudine [BVDU, (E)-5-(2-bromovinyl)-2'-deoxyuridine]. It has been confirmed that the catalytic rate of $D m-d N K$ for pyrimidines is 100-600-fold higher compared to purines (3). Taken together, these data underline the importance of its potential role as a suicide gene. Therefore, $D m-d N K$ has been used successfully in suicide gene therapy against a wide variety of cancers (4-8).

In terms of delivery vehicles, the efficacy of this approach may be further enhanced. For this purpose, in this study, we 
investigated recombinant vectors derived from replicationdefective adenovirus and lentivirus. Adenovirus is the most commonly used viral delivery tool for gene therapy. However, the major limitation in the application of this vector is the rapid inactivation of adenovirus from systemic delivery, thus losing the sustained gene expression (9-11). By contrast, lentiviral vectors may be incorporated into the host genome, thereby ensuring a prolonged gene expression with a limited host immune response (12). To date, the benefit of this therapy coupled with the replication-defective adenoviral gene for breast cancer remains to be elucidated.

The purpose of the present study was to compare the efficacy of replication-defective adenoviral and lentiviral gene therapy for breast cancer. The data presented in this study will provide a theoretical reference for future studies on cancer therapy.

\section{Materials and methods}

Cell line and cell culture. The Bcap37 human breast cancer cell line was obtained from the Cancer Institute of the China Medical University (Shenyang, China) and cultured in RPMI-1640 medium (Invitrogen Corp., Carlsbad, CA, USA) supplemented with $10 \%$ fetal bovine serum, $100 \mathrm{U} / \mathrm{ml}$ penicillin and $100 \mu \mathrm{g} / \mathrm{ml}$ streptomycin at $37^{\circ} \mathrm{C}$ in a $5 \% \mathrm{CO}_{2}$ incubator.

Construction of viral vectors. The coding sequence of Dm-dNK was released from plasmid PGEM-T-dNK with endonucleases EcoRI and BamHI (New England Biolabs, Beverley, MA) and cloned in-frame into plasmid Pentr13 [containing the sequence of a mouse CMV promoter, His-tag(N) and multiple cloning sites] and plasmid PGC-FU [Genechem, Shanghai, China; containing the sequence of a mouse CMV promoter, multiple cloning sites and a green fluorescent protein (GFP) sequence] to generate PENTR13-dNK and PGC-FU-dNK, respectively. The fragment expression cassette of $\mathrm{dNK}$ containing a mouse CMV promoter, His-tag(N) and the dNK gene from plasmid PENTR13-dNK were excised and inserted into plasmid pShuttle-basic (Sinogenomax, Inc., Beijing, China) to generate shuttle plasmid pShuttle-basic-His-tag(N)-dNK (plasmid Ad-CMV-dNK). The fragment expression of GFP from plasmid PGC-FU-dNK was removed with endonucleases AgeI and $E c o$ RI (NEB, UK) to generate the recombinant plasmid PGC-FU-CMV-dNK (plasmid Lenti-CMV-dNK). Plasmids PGC-FU, Ad-CMV-dNK and Lenti-CMV-dNK were individually transfected into the human embryonic kidney HEK293 cells using Lipofectamine ${ }^{\mathrm{TM}} 2000$ reagent (Invitrogen Corp.) according to the manufacturer's instructions. After homologous recombination, we obtained a replication-deficient adenovirus known as Ad-CMV- $\mathrm{dNK}$ and 2 lentiviruses known as Lenti-GFP and Lenti-CMV-dNK. The lentivirus and replication-deficient adenovirus harboring GFP alone (Lenti-GFP and Ad-GFP) driven by the CMV promoter were used to determine the infection rate of the Bcap37 cells.

Fluorescent-activated cell sorting (FACS) analysis. The Bcap37 breast caner cells were seeded in 6-well plates in $2 \mathrm{ml}$ of growth medium at a population of $3 \times 10^{5}$ cells/well. After culturing for $24 \mathrm{~h}$, Ad-GFP and Lenti-GFP were added to the medium at a multiplicity of infection (MOI) of 1 and 10 to achieve the gene transfer efficiency. Three days later, all cells were harvested and suspended in PBS with $0.5 \%$ bovine serum albumin at the population of $1 \times 10^{6}$ cells $/ \mathrm{ml}$. The samples were used to detect the expression profile of GFP by a FACScan flow cytometer equipped with CellQuest and Modfit LT for Mac V1.01 software (Becton-Dickinson, San Jose, CA).

$R T$-PCR. Bcap37 cells were seeded in 6-well plates at a density of $10^{5}$ cells/well for $24 \mathrm{~h}$. Subsequently, $D m-d N K$ transduced by different viruses at a MOI of 10 was added to the cell cultures. Polybrene $(6 \mu \mathrm{g} / \mathrm{ml})$ was added to all cultures. Total RNA was isolated from the cultured cells using TRIzol reagent (Sigma, USA) after 3 days of infection. The cDNA was synthesized from 1 ng of RNA using an RT-PCR kit (Takara, Japan) following the manufacturer's instructions. The primer sequences were as follows: sense, 5'-CCG GAA TTC ACC ATG GAG GCA-3' and antisense, 5'-CGC GGA TCC TCA TTA TCT GGC GAC-3' for $D m-d N K$ (779 bp); sense, 5'-ACC ACA GTC CAT GCC ATC AC-3' and antisense, 5'-TCC ACC ACC CTG TTG CTG TTG CTG TA-3' for GAPDH (452 bp). The PCR conditions were as follows: a pre-heating step at $94^{\circ} \mathrm{C}$ for $4 \mathrm{~min}, 35$ cycles with denaturing at $94^{\circ} \mathrm{C}$ for $1 \mathrm{~min}$, annealing at $60^{\circ} \mathrm{C}\left(55^{\circ} \mathrm{C}\right.$ for GAPDH) for $1 \mathrm{~min}$ and an extension at $72^{\circ} \mathrm{C}$ for $1.5 \mathrm{~min}$. PCR products were electrophoresed on a $2 \%$ agarose gel and the optical density of the bands was determined. The PCR product of the GAPDH gene was used as the internal control.

Activity assays of $D m-d N K$. In order to assess the function of $D m-d N K$ transduced by different viruses, we prepared cell extracts as previously described (13) from Bcap37 cells at $72 \mathrm{~h}$ after viral infection at a MOI of 10 . The activity of $D m-d N K$ was determined by DE-81 filter paper assay using tritiumlabeled substrates. Briefly, the assay was conducted in a $35-\mathrm{ml}$ reaction mixture containing $50 \mathrm{mM}$ Tris- $\mathrm{HCl}$ at $\mathrm{pH} 7.6,5 \mathrm{mM}$ $\mathrm{MgCl}_{2}, 2 \mathrm{mM}$ dithiothreitol, $15 \mathrm{mM} \mathrm{NaF}, 100 \mathrm{mM} \mathrm{KCl}, 5 \mathrm{mM}$ ATP, $0.5 \mathrm{mg} / \mathrm{ml} \mathrm{BSA}$ and $0.6 \mathrm{mg}$ protein extract. Equivalent amounts of unlabeled substrates were mixed with $2.5 \mathrm{mM}$ [methyl- ${ }^{3} \mathrm{H}$ ]dThd (Moravek Biochem, CA, USA) and incubated at $37^{\circ} \mathrm{C}$ for 10,20 and $30 \mathrm{~min}$. Subsequently, aliquots of the reaction mixtures were applied to Whatman DE-81 filter paper disks and dried for $1 \mathrm{~h}$. After washing 3 times with $5 \mathrm{mM}$ ammonium formate, the filter bound with nucleoside monophosphates was eluted with $0.5 \mathrm{M} \mathrm{KCl}$, and the radioactivity was quantified using a scintillation counter.

Cell viability and proliferation analysis. Exponentially growing Bcap37 cells were plated into 96-well plates $\left(10^{4}\right.$ cells/well $)$ and incubated for $24 \mathrm{~h}$. The cells were then infected with Lenti-GFP, Ad-CMV-dNK and LentiCMV-dNK at a MOI of 10. Post-transfection, the medium was treated with BVDU at various concentrations ranging from 0.001 to $10 \mu \mathrm{M}$ for $72 \mathrm{~h}$. Prior to measurement, a volume of $20 \mu \mathrm{l}$ of $5 \mathrm{mg} / \mathrm{ml}$ tetrazolium salt 3-(4,5-dimethylthiazol2-yl)-2,5-diphenyl tetrazolium bromide (MTT) (Promega, Madison, WI) in phosphate-buffered saline (PBS) was added to each well. After $4 \mathrm{~h}$ of incubation, the culture medium was removed and $200 \mu \mathrm{l}$ of dimethyl sulphoxide (DMSO) were added to dissolve the purple crystal followed by vibration for $10 \mathrm{~min}$. The absorbance was determined at $570 \mathrm{~nm}$ to evaluate 
the viable cells. Each experiment was performed in triplicate. In order to determine whether the cells transfected with different viruses were functional, we conducted proliferation assays in vitro. Briefly, Bcap37 cells transduced by Lenti-GFP, Ad-CMV-dNK and Lenti-CMV-dNK at a MOI of 10 were seeded to 24-well plates with a pre-coating of BVDU $(1 \mu \mathrm{M})$. During the incubation, cells were trypsinized and suspended in a serum-free medium. Cell counting was conducted under a microscope using a hemocytometer.

Cell apoptosis assay. Quantitative assessment of apoptosis was conducted by flow cytometry using the Annexin V-FITC/ propidium iodide (PI) double staining kit (Genmed Biosciences, China) according to the manufacturer's instructions. Following transfection and treatment with BVDU, Bcap37 cells were harvested, washed with PBS and resuspended in $200 \mu \mathrm{l}$ of binding buffer. A volume of $5 \mu \mathrm{l}$ of Annexin V-FITC and $10 \mu \mathrm{l}$ of PI was added and the mixture was incubated for $15 \mathrm{~min}$ in the dark. The apoptotic cells were analyzed by a FACSCalibar equipped with CellQuest and Modfit LT for Mac V1.01 software (Becton-Dickinson).

In vivo experiment. Female $\mathrm{BALB} / \mathrm{C}$ nude mice 6-7 weeks old were purchased from the Experimental Animal Center, Chinese Academy of Sciences (Shanghai, China). All animals were handled strictly in compliance with the Guidelines for the Care and Use of Laboratory Animals (National Research Council, 1996). The mice were inoculated subcutaneously in the flank with $1.0 \times 10^{7}$ Bcap37 cells. When the average volume of tumors reached $\sim 100 \mathrm{~mm}^{3}$, the mice were randomly divided into 4 groups (6 mice/group) as follows: i) BVDU with PBS; ii) BVDU with Lenti-GFP; iii) BVDU with Ad-CMV-dNK and iv) BVDU with Lenti-CMV-dNK. The mice were injected intratumorally with the viruses at a dose of $10^{9}$ pfu 3 times with a 2-day interval. Subsequently, $5 \mathrm{mg} / \mathrm{kg}$ of BVDU was administered daily into the peritoneal cavity for 7 consecutive days. Tumor growth was monitored every 5 days and the dimension of the tumors was recorded with a caliper for up to 30 days.

Statistical analysis. The data are expressed as the means \pm SD and analyzed using the statistical software SPSS (version 10.1, Chicago, IL). $\mathrm{P}<0.05$ was considered to indicate a statistically significant difference.

\section{Results}

$D m-d N K$ expression. Dm- $d N K$-expressed replicationdefective adenoviral and lentiviral vectors were successfully constructed in the $293 \mathrm{~T}$ cells. Both expression cassettes contained a CMV promoter required to drive the expression of the $D m-d N K$ gene (Fig. 1A). The titers of both types of viruses were $2 \times 10^{11}$ and $2 \times 10^{9} \mathrm{TU} / \mathrm{ml}$, respectively. The infection efficiency was assessed by transducing the Bcap37 human breast cancer cell line with adenoviral and lentiviral vectors carrying the GFP construct (Fig. 1B). Compared with the vector infection at a MOI of 1, a significantly higher population ( $90-95 \%)$ of GFP-positive cells was observed under the condition of a vector infection at a MOI of 10 . Moreover, both vectors were efficiently transduced in the Bcap37 cell line at a MOI of 10 .
Therefore, a MOI of 10 was the optimal dose for the transfection of Bcap37 cells. Furthermore, no significant difference in the infection efficiency at a MOI of 10 between both types of vectors was observed; therefore, Lenti-GFP was selected as the representative for the following studies. Similarly, as demonstrated in Fig. 1C, GFP expression was stronger at a MOI of 10 in the Bcap37 cells.

The expression of $D m-d N K$ was also evaluated by RT-PCR. Bcap37 cells transduced with Ad-CMV-dNK and Lenti-CMV-dNK revealed a significantly higher expression of Dm-dNK mRNA (Fig. 2). Moreover, the phosphorylation of dThd in cell extracts was examined in order to evaluate the activity of $D m-d N K$ when imported into cancer cells. The activity of $D m-d N K$ in the Bcap37 cells transduced with Ad-CMV-dNK and Lenti-CMV-dNK revealed a 3-fold increase compared to that in the cells transduced with Lenti-GFP (Fig. 3), with a significant difference $(\mathrm{P}<0.05)$, although no obvious difference $(\mathrm{P}>0.05)$ between the cells transduced with Ad-CMV-dNK and Lenti-CMV-dNK was observed.

Cell cytotoxicity, proliferation and induction of apoptosis. Bcap37 cells were transduced with Lenti-GFP, Ad-CMV-dNK and Lenti-CMV-dNK at a MOI of 10 and incubated with BVDU at increasing concentrations for $72 \mathrm{~h}$, followed by viability analysis using MTT assay. Cytotoxicity was observed in the Bcap37 cells infected with Ad-CMV-dNK and LentiCMV-dNK in the presence of BVDU (Fig. 4A). The survival rates of the Bcap37 cells transduced with Ad-CMV-dNK and Lenti-CMV-dNK in the presence of $1 \mu \mathrm{M}$ BVDU were $\sim 55.3$ and $49.7 \%$, respectively. A more prominent cytotoxic effect was observed in the presence of high concentrations of BVDU, suggesting that the viability of the cells revealed a decreasing trend in a dose-dependent manner. Moreover, the Bcap37 cells treated with both Ad-CMV-dNK and LentiCMV-dNK exhibited a decreasing trend in viability when compared with the control or with the cells treated with Lenti-GFP. The proliferation of the transduced Bcap37 cells in the presence of $1 \mu \mathrm{M}$ BVDU was further analyzed. Both Ad-CMV-dNK and Lenti-CMV-dNK revealed an apparent inhibitory effect on cell growth when compared with the Lenti-GFP group after $72 \mathrm{~h}(\mathrm{P}<0.05)$ (Fig. 4B) . However, no significant difference between the Ad-CMV-dNK and LentiCMV-dNK groups was observed ( $\mathrm{P}>0.05)$.

In an attempt to gain further insight into the possible mechanisms of this suicide gene therapy, FACS analysis was used to evaluate the apoptosis of Bcap37 cells. Ad-CMV-dNK and Lenti-CMV-dNK resulted in higher apoptotic rates of $63.5 \pm 5.8$ and $73.1 \pm 6.8 \%$, respectively, at a BVDU dose of $1 \mu \mathrm{M}$ (Fig. 5). Conversely, the apoptotic rate was only $13.8 \pm 2.1 \%$ in the cells transduced with Lenti-GFP, indicating that $D m-d N K$ had a slight contribution to the cytotoxicity. Furthermore, there was no difference in apoptosis between the Ad-CMV-dNK and Lenti-CMV-dNK groups ( $\mathrm{P}>0.05)$, which was consistent with its expression level in the previous experiment, thus establishing a connection between the apoptotic level and the activity of $D m-d N K$ in the experimental cell line.

Antitumor efficacy of Dm-dNK in vivo. The therapeutic efficacy of lentiviral and adenoviral vectors encoding a targeted 
A

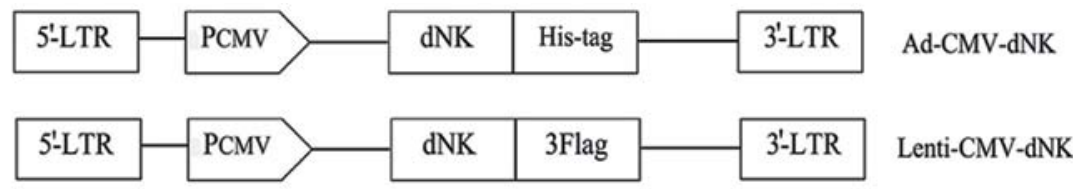

B

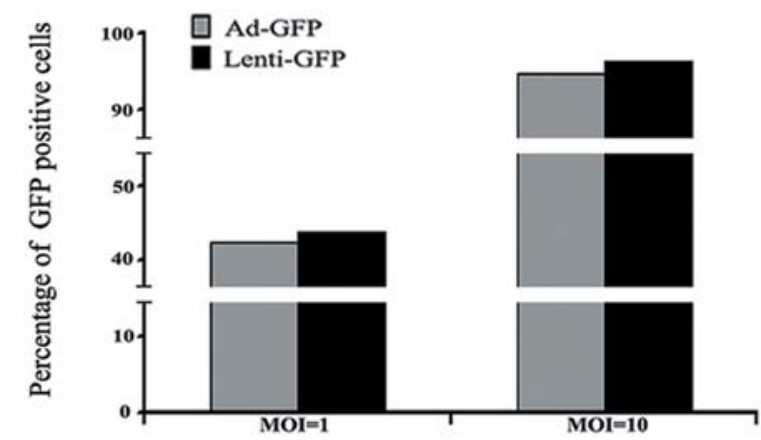

C

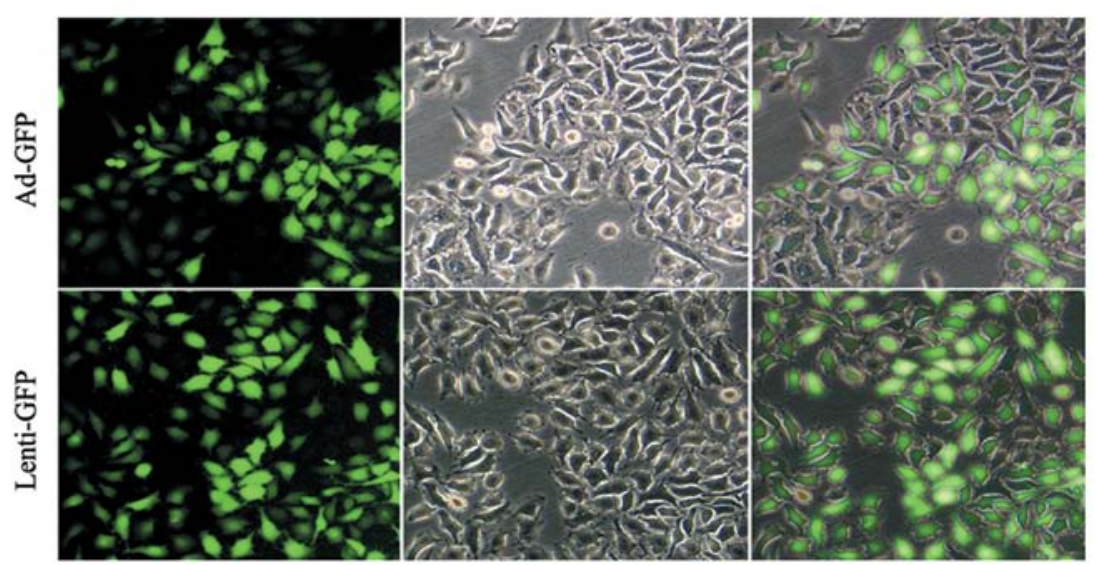

Figure 1. Expression cassettes and infection rates of $D m-d N K$ and green fluorescent protein (GFP). (A) Structural illustration of $D m$ - $d N K$ delivered by the replication-defective adenoviral and lentiviral vectors, respectively. Both therapeutic expression cassettes contained a CMV promoter required to drive the expression of the $D m-d N K$ gene. LTR, long terminal repeat; CMV, cytomegalovirus promoter. (B) Bcap37 cells were infected with Ad-GFP and Lenti-GFP at MOIs of 1 and 10. After 3 days, GFP-positive cells were detected using FACS analysis. More than 90\% of the cells were transduced with Ad-GFP and Lenti-GFP at a MOI of 10. Data derived from a single experiment are represented. (C) GFP expression was observed in the Bcap37 cells transduced with Ad-GFP and Lenti-GFP at a MOI of 10 .

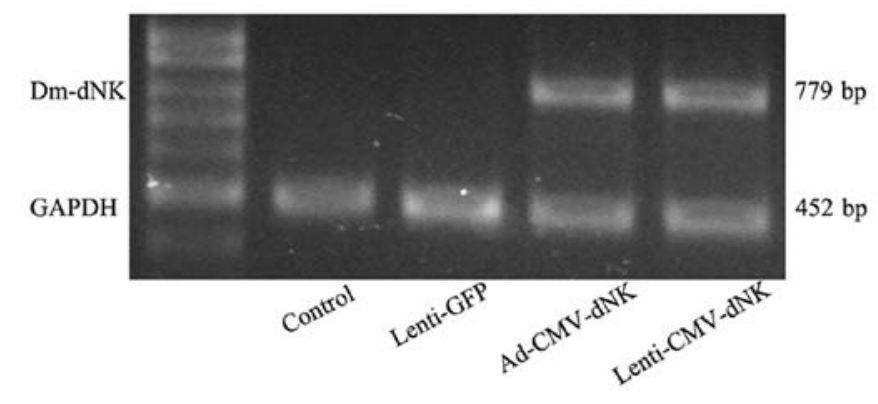

Figure 2. Expression of $D m-d N K$ mRNA in Bcap37 cells determined by RT-PCR. Dm- $d N K$ mRNA was highly expressed in Bcap37 cells infected with Ad-CMV-dNK and Lenti-CMV-dNK, while no expression was detected in cells infected with Lenti-GFP or PBS. The products with the expected sizes of 779 and 452 bp were observed in $D m-d N K$ and GAPDH transcripts, respectively.

gene in nude mice was compared, revealing a dramatic difference. A significant inhibitory effect on tumor growth

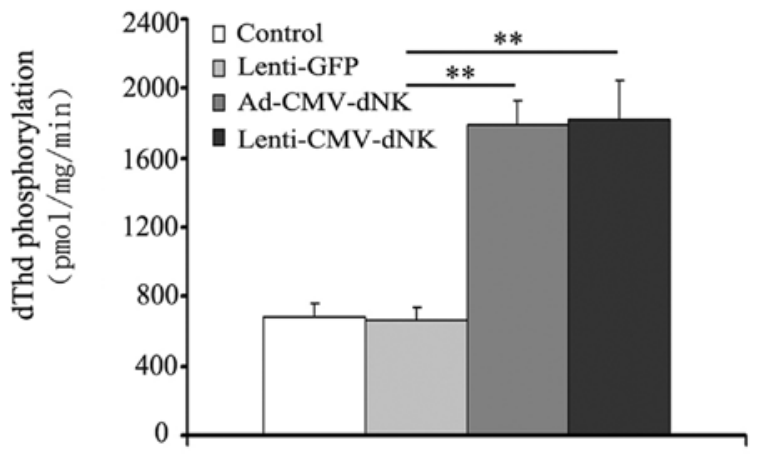

Figure 3. The activity of $D m-d N K$ phosphorylated by $\left[{ }^{3} \mathrm{H}\right] \mathrm{dThd}$ in transduced cells. Bcap37 cells infected with Ad-CMV-dNK and Lenti-CMV-dNK revealed 2.68- and 2.74-fold enhancement compared with the Lenti-GFP treated cells. ${ }^{* *} \mathrm{P}<0.01$ vs. Lenti-GFP.

in either the Ad-CMV-dNK or Lenti-CMV-dNK groups combined with BVDU treatment when compared with the 

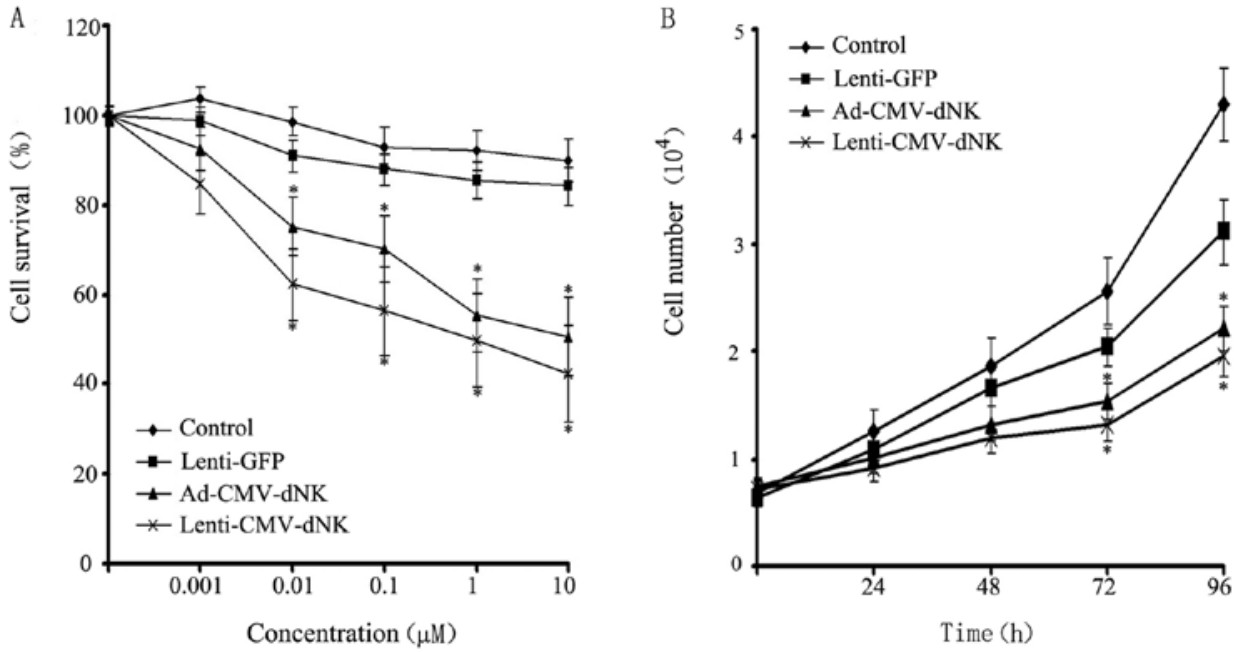

Figure 4. Cell viability and proliferation assay of $D m-d N K$ with BVDU treatment. (A) Cell viability assay of infected Bcap37 cells combined with BVDU. Bcap37 cells infected with Lenti-GFP, Ad-CMV-dNK and Lenti-CMV-dNK were treated with BVDU at various doses. MTT assay was used to detect cell viability. (B) Cell proliferation assay of infected Bcap37 cells combined with BVDU. Bcap37 cells infected with Lenti-GFP, Ad-CMV-dNK and LentiCMV-dNK at a MOI of 10 were seeded in 24-well plates with a pre-coating of $1 \mu \mathrm{M}$ BVDU. During incubation, cell counting was performed. Data are presented as the means $\pm \mathrm{SD}$ from 3 independent experiments. ${ }^{*} \mathrm{P}<0.05$ vs. Lenti-GFP.

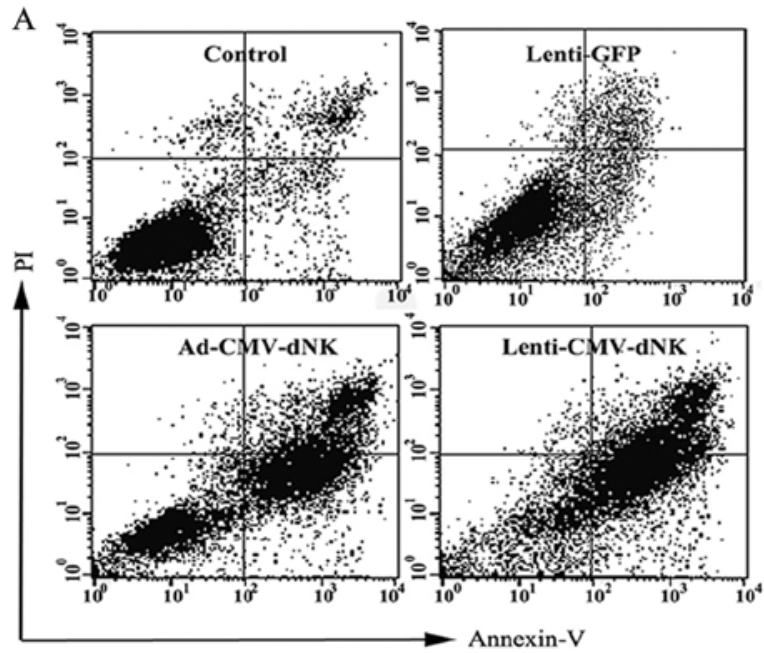

B

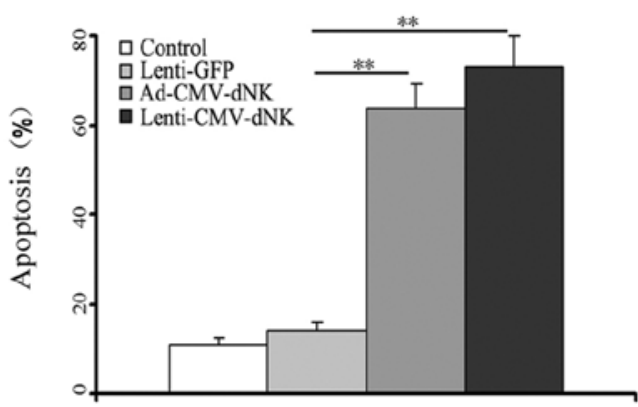

Figure 5. Analysis of apoptosis in virally transduced Bcap37 cells following exposure to BVDU. (A) The cells were collected and stained with Annexin V-FITC/PI and analyzed by flow cytometry. A significant induction of apoptosis was detected. (B) Results are representative of 3 independent experiments. ${ }^{* * *} \mathrm{P}<0.01$ compared with the Lenti-GFP group.

control or Lenti-GFP group combined with BVDU treatment was demonstrated (Fig. 6). The average volumes of the tumors from the control or Lenti-GFP injection group combined with BVDU treatment after 30 days were 1,023.48 \pm 191.39 and $967.79 \pm 81.21 \mathrm{~mm}^{3}$, respectively, which were much larger compared to those from either the Ad-CMV-dNK or Lenti-CMV-dNK group combined with BVDU treatment $\left(650.34 \pm 68.30\right.$ and $424.22 \pm 46.74 \mathrm{~mm}^{3}$, respectively) $(\mathrm{P}<0.05)$. Moreover, the average volume of tumors at day 30 from the Ad-CMV-dNK group combined with BVDU was larger compared to that from the Lenti-CMV-dNK group combined with BVDU $(\mathrm{P}<0.05)$. These findings indicate that lentiviral vectors may the improve the therapeutic benefit in long-term therapy in vivo.

\section{Discussion}

Despite several set-backs, gene-directed enzyme prodrug therapy (GDEPT) remains a promising approach for cancer gene therapy. This strategy has a significant advantage over conventional chemotherapy since it reduces the toxicity induced by the prodrug to target cells $(14,15)$. With the efforts to improve the therapeutic efficacy, viral vectors are useful for gene delivery. In particular, adenoviral and lentiviral vectors as highly effective gene carriers have been widely applied.

Adenoviral vectors are extensively utilized in a wide range of cancer cells. Adenoviruses retain their extrachromosomal form, transduce susceptible cells efficiently and produce high titers. However, the systemic delivery of adenoviruses causes 


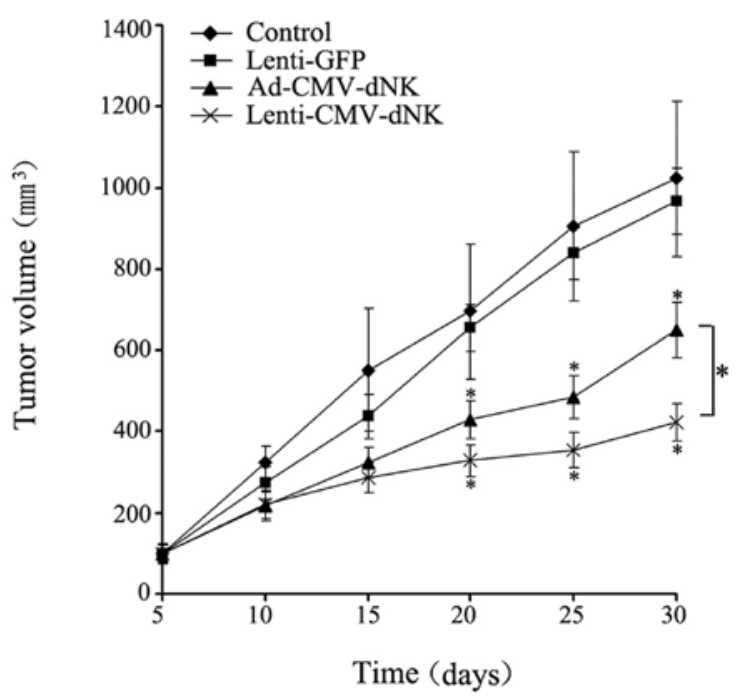

Figure 6. In vivo measurement of tumor growth. The cells were subcutaneously injected into nude mice. The length and width of tumors were measured every 5 days after injection. The volume of tumors was calculated and presented as the means \pm SD for all tumors in each group. Tumor volume was determined by following the equation of $1 / 2 \mathrm{x}$ length $\mathrm{x}$ width ${ }^{2} .{ }^{*} \mathrm{P}<0.05 \mathrm{vs}$. Lenti-GFP.

a variety of host immune responses, which strongly limits its safe application in vivo. Lentiviral vectors have advantages over adenoviral vectors since they are incorporated into the host genome to establish a stable transgene expression, which provides a promising role in improving therapeutic efficacy of gene therapy (16). The unique properties of both vectors provide the impetus for us to conduct experimental and clinical studies of cancer gene therapy. To date, to the best of our knowledge, there are no reports related to the therapeutic outcomes of adenoviral and lentiviral vectors expressing $D m-d N K$ in the gene therapy of cancer. In the present study, the adenovirusand lentivirus-mediated expression of $D m-d N K$ was observed to offer efficient treatment for human breast cancer. Moreover, lentivirus exhibited great potential for long-lasting expression of the transgene. Therefore, the vectors may be used in the majority of clinical cases.

Over the past decade, $D m-d N K$ has been reported to phosphorylate all precursor nucleosides. The deoxyribonucleoside kinase may be expressed in human cells and no difference in its cellular sensitivity to nucleoside analogs was observed when this enzyme was expressed in the cytosol or nucleus $(17,18)$. The broad substrate specificity of $D m-d N K$, as well as its high catalytic rate, makes it a promising candidate of the nucleoside kinase family. On the basis of previous findings (5-7), Dm- $d N K$ has been shown to exert potential antitumor activity. However, the underlying mechanism remains unclear. Current interest is focused on substrate specificity in the activation of nucleoside analogue to improve therapeutic efficiency. Knecht et al addressed the issue that a few amino acid substitutes may change the substrate specificity of $D m-d N K$ (19). Moreover, the deoxyribonucleoside kinase mutant exhibits a relatively increased sensitivity to nucleoside analogs and a simultaneous positive therapeutic effect in cancer cells $(20,21)$. Therefore, further studies are required to quantify interconversation rates of $D m-d N K$ and mutants and provide a more effective gene therapeutic strategy.
Knecht et al convincingly demonstrated that gemcitabine, 2',2'-difluoro-deoxycytidine $(\mathrm{dFdC})$, an anticancer drug, is an efficient substrate for $D m-d N K$, which may explain the structural basis for the interaction between $D m-d N K$ and gemcitabine (22). Nevertheless, the underlying mechanisms of other efficient prodrug candidates such as $1-\beta$-D-arabinofuranosylcytosine (AraC, cytarabine) remain an area of active investigation. Further studies are required to decipher the precise role(s) of the correlation between $D m-d N K$ and nucleoside analogs in cancer treatments combined with gene therapy.

For attempts to optimize the therapeutic efficacy, adenoassociated virus (AAV) as a promising mammalian virus vector is commonly used for gene therapy and is considered one of the safest viral vectors due to its stability as a vector genome and its persistent high expression level $(23,24)$. Compared with other viral vectors, AAV may elicit minimal immune responses. Recently, studies using animal models of stromal transduction with AAV vectors have shown the advantages of this vector $(25,26)$. However, it has been reported that AAV integration is associated with hepatocellular carcinoma (27). Clearly, additional research is required to more precisely elucidate these findings.

In conclusion, in this study, we demonstrate that replicationdefective adenoviral and lentiviral vectors highly expressing a suicide gene can be effectivley used in suicide gene therapy for the treatment of human breast cancer. However, the lentiviral vector demonstrates broad implications for the long-term expression of a therapeutic gene in the strategy of suicide gene therapy. By employing lentiviral vectors, the $D m-d N K / \mathrm{BVDU}$ system may provide a safe and effective modality for the treatment of breast cancer.

\section{Acknowledgements}

The present study was supported by grants from the Hi-Tech Research Development Program of China (863 Program, 2006AA02Z493) and National Natural Science Foundation of China (Nos. 81071900, 81272920 and 81172199).

\section{References}

1. Akcay MN: Metastatic disease in the breast. Breast 11: 526-528, 2002.

2. Munch-Petersen B, Piskur J and Sondergaard L: Four deoxynucleoside kinase activities from Drosophila melanogaster are contained within a single monomeric enzyme, a new multifunctional deoxynucleoside kinase. J Biol Chem 273: 3926-3931, 1998.

3. Munch-Petersen B, Knecht W, Lenz C, Sondergaard L and Piskur J: Functional expression of a multisubstrate deoxyribonucleoside kinase from Drosophila melanogaster and its C-terminal deletion mutants. J Biol Chem 275: 6673-6679, 2000.

4. Ito M, Suda Y, Harashima $\mathrm{H}$ and Kamiya $\mathrm{H}$ : Cytotoxic effect of Drosophila deoxynucleoside kinase gene on replicating plasmid in HeLa cells. Biol Pharm Bull 33: 1223-1227, 2010.

5. Zhu Z, Mao L, Zhao L, et al: Synergistic therapeutic effect in gastric cancer cells produced by oncolytic adenovirus encoding Drosophila melanogaster deoxyribonucleoside kinase. Cancer Biol Ther 11: 874-882, 2011.

6. Ma S, Zhao L, Zhu Z, et al: The multisubstrate deoxyribonucleoside kinase of Drosophila melanogaster as a therapeutic suicide gene of breast cancer cells. J Gene Med 13: 305-311, 2011.

7. Ma S, Qu W, Mao L, et al: Antitumor effects of oncolytic adenovirus armed with Drosophila melanogaster deoxyribonucleoside kinase in colorectal cancer. Oncol Rep 27: 1443-1450, 2012. 
8. Zhang N,Zhao L, Ma S, Gu M and Zheng X: Lentivirus-mediated expression of Drosophila melanogaster deoxyribonucleoside kinase driven by the hTERT promoter combined with gemcitabine: a potential strategy for cancer therapy. Int J Mol Med 30: 659-665, 2012.

9. Kojaoghlanian T, Flomenberg P and Horwitz MS: The impact of adenovirus infection on the immunocompromised host. Rev Med Virol 13: 155-171, 2003.

10. Poller W, Fechner H, Noutsias M, Tschoepe C and Schultheiss HP Highly variable expression of virus receptors in the human cardiovascular system. Implications for cardiotropic viral infections and gene therapy. Z Kardiol 91: 978-991, 2002.

11. VandenDriessche T, Collen D and Chuah MK: Viral vectormediated gene therapy for hemophilia. Curr Gene Ther 1: 301-315, 2001.

12. Sugiyama O, An DS, Kung SP, et al: Lentivirus-mediated gene transfer induces long-term transgene expression of BMP-2 in vitro and new bone formation in vivo. Mol Ther 11: 390-398, 2005.

13. Sandrini MP, Clausen AR, On SL, Aarestrup FM, MunchPetersen B and Piskur J: Nucleoside analogues are activated by bacterial deoxyribonucleoside kinases in a species-specific manner. J Antimicrob Chemother 60: 510-520, 2007.

14. Bonini C, Bondanza A, Perna SK, et al: The suicide gene therapy challenge: how to improve a successful gene therapy approach. Mol Ther 15: 1248-1252, 2007.

15. Yazawa K, Fisher WE and Brunicardi FC: Current progress in suicide gene therapy for cancer. World J Surg 26: 783-789, 2002

16. Bastide C, Maroc N, Bladou F, et al: Expression of a mode gene in prostate cancer cells lentivirally transduced in vitro and in vivo. Prostate Cancer Prostatic Dis 6: 228-234, 2003.

17. Johansson M, Brismar S and Karlsson A: Human deoxycytidine kinase is located in the cell nucleus. Proc Natl Acad Sci USA 94 11941-11945, 1997
18. Zhu C, Johansson M and Karlsson A: Incorporation of nucleoside analogs into nuclear or mitochondrial DNA is determined by the intracellular phosphorylation site. J Biol Chem 275: 26727-26731, 2000.

19. Knecht W, Sandrini MP, Johansson K, Eklund H, MunchPetersen B and Piskur J: A few amino acid substitutions can convert deoxyribonucleoside kinase specificity from pyrimidines to purines. EMBO J 21: 1873-1880, 2002.

20. Solaroli N, Bjerke M, Amiri MH, Johansson M and Karlsson A: Active site mutants of Drosophila melanogaster multisubstrate deoxyribonucleoside kinase. Eur J Biochem 270: 2879-2884, 2003.

21. Zhu Z, Ma S, Zhao L, et al: Adenovirus-mediated Drosophila melanogaster deoxyribonucleoside kinase mutants combined with gemcitabine harbor a safe cancer treatment profile. Int J Oncol 38: 745-753, 2011.

22. Knecht W, Mikkelsen NE, Clausen AR, et al: Drosophila melanogaster deoxyribonucleoside kinase activates gemcitabine. Biochem Biophys Res Commun 382: 430-433, 2009.

23. Mueller $\mathrm{C}$ and Flotte TR: Clinical gene therapy using recombinant adeno-associated virus vectors. Gene Ther 15: 858-863, 2008.

24. Calcedo R, Vandenberghe LH, Gao G, Lin J and Wilson JM: Worldwide epidemiology of neutralizing antibodies to adenoassociated viruses. J Infect Dis 199: 381-390, 2009.

25. Liu J, Saghizadeh M, Tuli SS, et al: Different tropism of adenoviruses and adeno-associated viruses to corneal cells: implications for corneal gene therapy. Mol Vis 14: 2087-2096, 2008.

26. Sharma A, Tovey JC, Ghosh A and Mohan RR: AAV serotype influences gene transfer in corneal stroma in vivo. Exp Eye Res 91: 440-448, 2010.

27. Donsante A, Miller DG, Li Y, et al: AAV vector integration sites in mouse hepatocellular carcinoma. Science 317: 477, 2007. 